The $8^{\text {th }}$ International Conference of the Slovenian Society for Non-Destructive Testing

"Application of Contemporary Non-Destructive Testing in Engineering"

September 1-3, 2005, Portorož, Slovenia, pp. 189-197

\title{
STUDIES OF RADIATION EMBRITTLEMENT OF MODEL ALLOYS BY THERMOELECTRIC MEASUREMENTS AND POSITRON ANNIHILATION SPECTROSCOPY
}

\author{
B. Acosta ${ }^{1}$, A. Zeman $^{2}$, L. Debarberis ${ }^{1}$ \\ ${ }^{1}$ European Commission, Joint Research Centre, Institute for Energy \\ Westerduinweg 3,1755 LE Petten, The Netherlands, \\ E-mails: beatriz.acosta-iborra@jrc.nl, Luigi.DEBARBERIS@cec.eu.int \\ ${ }^{2}$ Slovak University of Technology, Department of Nuclear Physics and Technology \\ Ilkovičova 3, 81219 Bratislava, Slovakia, \\ Fellow at European Commission, Joint Research Centre, Institute for Energy \\ Westerduinweg 3, 1755 LE Petten, The Netherlands, \\ E-mail: andrej.zeman@jrc.nl
}

\begin{abstract}
One of the basic mechanisms of radiation embrittlement of steels and welds is due to matrix damage. Embrittlement results in a raise in the ductile-to-brittle transition temperature, which is normally used as indicator of the degradation status of the material. Other methods different than mechanical tests can be used to follow embrittlement like small punch test, non-destructive measurements of thermo-electric and magnetic properties, positron annihilation spectroscopy (PAS) measurements, etc. As regards assessment of reactor pressure vessel's radiation embrittlement, a need exists for correlation not only between the microstructural changes and the non-destructive techniques (NDT) results, but also between the non-destructive evaluation (NDE) outcomes and the mechanical behaviour or level of embrittlement.

At the JRC-IE the measurement of thermoelectric voltage using a specially developed nondestructive method (STEAM) to assess the embrittlement state of materials is regularly performed. The positron annihilation spectroscopy in lifetime setup (PAS-LT) is used for study the microstructural changes of matrix due to embrittlement process. Both techniques, STEAM and PAS, have been applied to test a number of complex model alloys with different chemical composition, and several irradiation conditions.

The obtained results prove that both techniques are good indicators of the change in material properties, given that their main parameter increases by effect of radiation dose like it occurs with the ductile-to-brittle transition temperature. Furthermore the combination of thermoelectric "STEAM" and PAS measurements seems to be promising for investigation of irradiation degradation mechanisms.
\end{abstract}

Keywords: Thermo-electric properties, Positron annihilation spectroscopy, Model alloys, Reactor pressure vessel materials, Irradiation embrittlement, Alloying elements 


\section{Introduction}

The main today's objective from a nuclear-industry perspective is to maximize and enhance the safety and the reliability of nuclear power plants (NPP) and to extend their operation lifetime. From this point of view the reactor pressure vessel (RPV) is one of the critical elements, rated as the highest priority category 1 component in all different national safety rankings. Being practically and economically un-replaceable, the RPV is subjected to significant usage and degradation and often becomes the component determining the operational safety of the NPPs. The degradation mechanism of RPV steel is a combination of very complex processes dependent on many factors, as the chemical compositions of the materials, the level of impurities, the radiation exposure as well as the thermal and mechanical treatments.

The properties of the RPV steels and the influence of thermal and neutron treatment on these properties are routinely investigated by destructive methods (surveillance programmes). The most important effect of the degradation by radiation in steels and welds is the decrease in their ductility. The main ways to determine the mechanical behaviour of such steels are tensile and Charpy impact tests, from which the ductile to brittle transition temperature (DBTT) and its increase due to neutron irradiation can be calculated. These tests are destructive and regularly applied to assess the integrity of structural materials. Beside standard mechanical testing methods used for structural integrity analysis of materials, in the last decade non-destructive techniques (NDT) are also applied for microstructural evaluation studies the more and more widely.

Few semi-empirical laws, based on macroscopic data, have been established, but such laws are not completely consistent with all data and do not always provide the desired accuracy. Because of the fact that key issues for primary components of NPPs (as, verification of structural integrity with focus to the RPV, prediction of degradation mechanisms and monitoring of ageing processes) are still on-going within the frame of SAFELIFE action of the JRC, many additional test methods, have therefore been developed to unravel the complex microscopic mechanisms responsible for RPV steel embrittlement.

The possibility of applying validated non-destructive monitoring techniques would however facilitate the surveillance of such materials.

\section{Neutron embrittlement mechanisms}

The key embrittlement mechanisms taking place during neutron irradiation of reactor pressure vessel (RPV) steels and welds [1] have been analysed in several papers [2-5,7]. General agreement exists on three basic mechanisms contributing to neutron embrittlement (occurring before vessel annealing) for both steels and welds: direct matrix damage, irradiation induced precipitation and elements segregation. In spite of this fact the models for analysis of radiation embrittlement are mainly based on statistical correlation of large sets of data.

The contribution of the various alloying elements and impurities and their interactions to irradiation hardening and embrittlement is not yet fully comprehended and several projects aimed to the understanding and simulating such irradiation degradation mechanism are still ongoing in particular, nickel content seems to confirm the hypothesis that it affects both the size (decreases) and distribution (more homogeneous) of the $\mathrm{Cu}$-rich clusters (or precipitates). Nickel also takes part in the composition of these clusters, as well as of the P-rich ones. The formation of solute clusters during irradiation of RPV steels results in depletion of $\mathrm{Cu}$ and $\mathrm{P}$ in the matrix; investigations showed that $\mathrm{Ni}$ delays this depletion. The effect of manganese seems to be important too, because it could accelerate the sensitivity of other key alloying elements from the radiation stability point of view. 
As neutrons interact with the crystalline structure of the steel, the ferrite matrix is directly damaged. In a given material, temperature and stress state matrix damage can be assumed to be simply dependent on fluence. At higher irradiation temperatures, the rate of damage is considered to be decreasing due to increased mobility of atoms and vacancies enhancing recombination processes. During matrix damage formation, copper, together with other elements, is known to lead precipitation of nano-precipitates also inducing matrix hardening and embrittlement. This precipitation effect is expected to saturate due to the progressive reduction of available precipitable elements in solid solution; in particular with the copper concentration. In addition, other elements, like $\mathrm{P}$, can segregate inside the grains (i.e. at dislocation planes), interacting with matrix defects (i.e. vacancies, interstitials, dislocation loops) or may be attracted to the $\mathrm{Cu}$-type precipitates. Phosphorus also migrates to grain boundary through diffusion processes $[6,7,8]$.

\section{Results from neutron irradiated RPV model alloys}

Experimental work to develop NDT methods to monitor degradation of materials is carried out within the frame of the SAFELIFE action of the JRC and it is oriented to the investigation of RPV model alloys with following main objectives:

(a) to study the contribution of the various alloying elements and their interactions to irradiation hardening and embrittlement,

(b) to verify the effect of alloying elements; in particular to verify the effect to the size and distribution of the radiation induced defects,

(c) to evaluate destructive and non-destructive outcomes based on correlation between macroscopic and microscopic parameters.

A matrix of 32 different complex RPV model alloys have been irradiated in the High Flux Reactor (HFR) in order to study systematically the effect of copper, phosphorus and nickel on irradiation embrittlement. The alloys have a common basic chemical composition and a parametric variation of $\mathrm{Cu}, \mathrm{P}$ and $\mathrm{Ni}$ contents. The most important results have been already published on different peer reviewed magazines; including results on thermoelectric voltage measurements (STEAM), positron annihilation spectroscopy (PAS) and magnetic testing (MBT) [9]. An example of positron annihilation Doppler broadening (PADB) results obtained by AEA technology is given in Fig. 1.

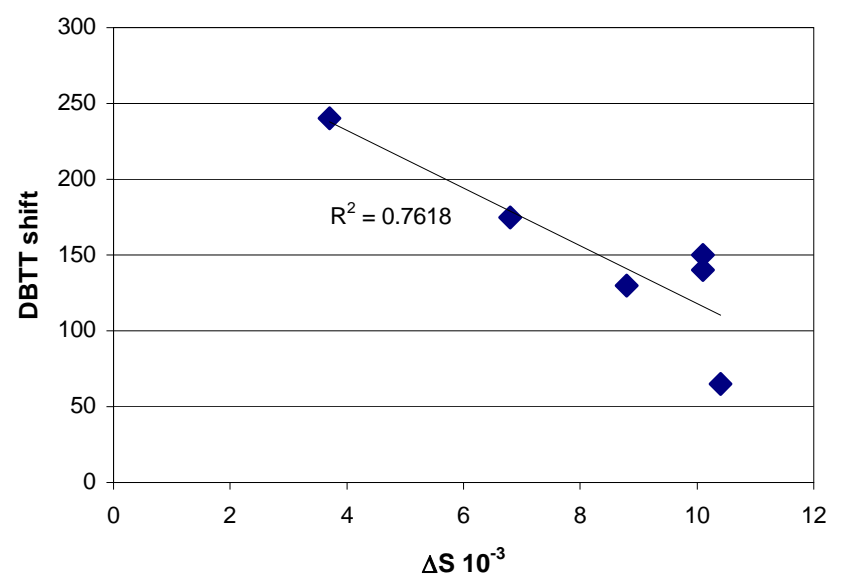

Fig. 1: Correlation is observed on positron annihilation parameters changes $(\Delta S)$ and ductile-tobrittle transition temperature shift for RPV model alloys.

A significant correlation is observed on PA parameters changes (line-shape parameter, $\mathrm{S}$, in this case) and mechanical properties degradation due to neutron embrittlement. For Fig. 1 six 
different model alloys before and after irradiation have been considered. Measurements on the same model alloys also show a strong correlation between positron annihilation and thermoelectric voltage measurements. This is shown in Fig. 2 where the variation of the Relative Seebeck Coefficient (RSC) obtained with the STEAM device is plotted against the S parameter.

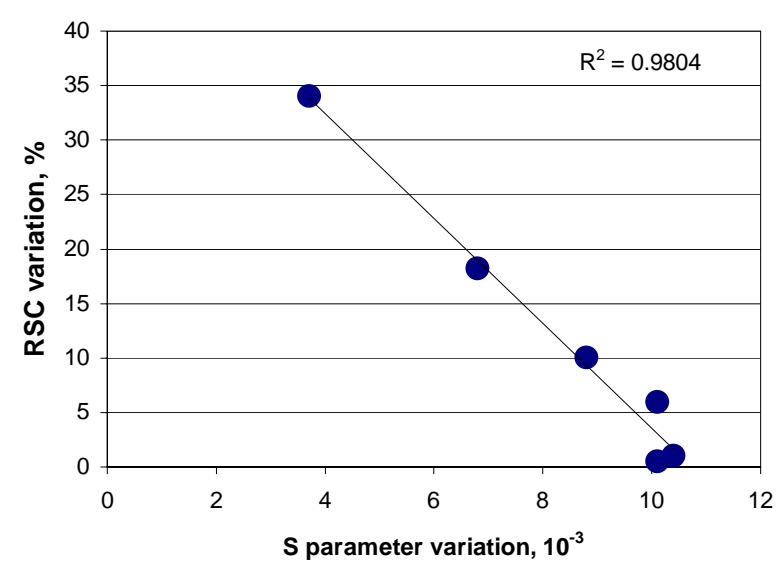

Fig. 2: Correlation between S-parameter and RSC variations for RPV model alloys.

Other interesting potential of thermoelectric measurement to follow copper precipitation in high nickel steels have been recently showed [10]. According to the precipitation model, $\mathrm{Cu}$ is expected to decrease during irradiation with the effect of increasing the value of the RSC. As it can be seen in Fig. 3, the RSC values for the high $\mathrm{Cu}$ alloys $(\approx 0.4 \mathrm{wt} . \%)$ are consistently increasing after irradiation; approaching RSC values of the un-irradiated alloys with $\approx 0.1 \mathrm{wt} . \%$ $\mathrm{Cu}$.

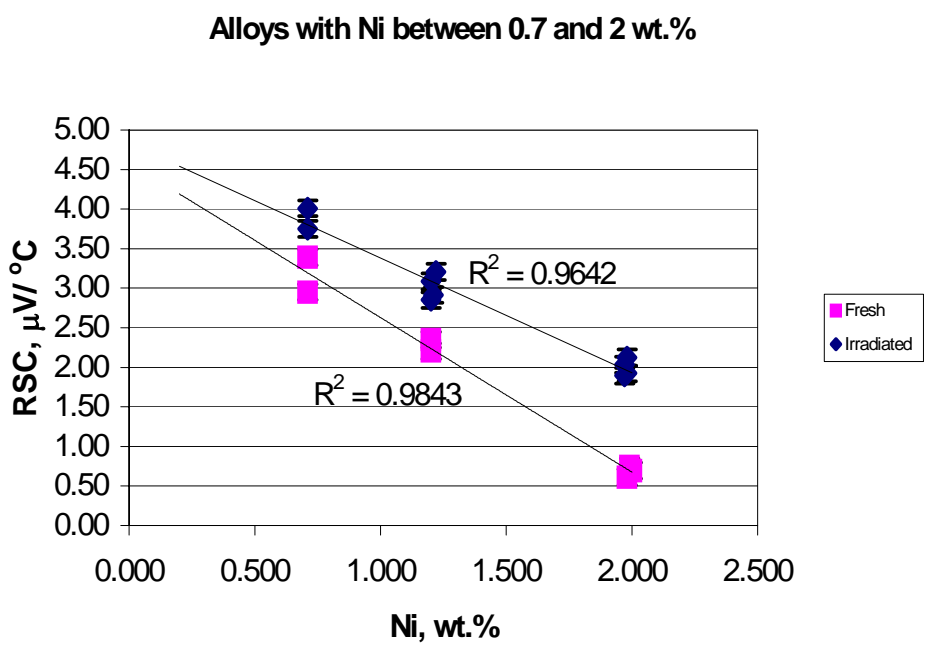

Fig. 3: RSC as function on $\mathrm{Ni}$, before and after irradiation, initial $\mathrm{Cu}$ content $\approx 0.4 \mathrm{wt} \%$

Further decrease of $\mathrm{Cu}$ content below a certain threshold in concentration does not affect the RSC value significantly. For the analysed sets of model alloys such threshold occurs at $\approx 0.08$ wt.\% Cu. Above this threshold, for high nickel alloys, the changes in RSC are then following the decrease of copper available in the matrix, hence following $\mathrm{Cu}$ precipitation effects. It is also noticed that larger variations of RSC for the same copper content take place for higher Ni contents, as Fig. 3 shows. 


\section{Results from proton irradiated RPV model alloys}

Recently three RPV model alloys with different alloying element's content were selected for investigation after proton irradiation; the data of such alloys are given in Table 1 .

Table 1: Chemical composition and mechanical properties of RPV model alloys.

\begin{tabular}{|c|c|c|c|c|c|c|}
\hline Sample & $\begin{array}{c}\text { Cu wt. } \\
\text { \% }\end{array}$ & $\begin{array}{c}\text { Ni wt. } \\
\text { \% }\end{array}$ & $\begin{array}{c}\text { P wt. } \\
\text { \% }\end{array}$ & $\begin{array}{c}\text { Si wt. } \\
\text { \% }\end{array}$ & $\begin{array}{c}\text { Mn wt. } \\
\text { \% }\end{array}$ & $\begin{array}{c}\text { DBTT } \\
{ }^{\circ} \mathbf{C}\end{array}$ \\
\hline MA-635 & 0.005 & 0.007 & 0.029 & 0.52 & 0.5 & -61.92 \\
\hline MA-440 & 0.4 & 0.71 & 0.002 & 0.17 & 0.47 & -84.05 \\
\hline MA-175 & 0.11 & 1.14 & 0.01 & 0.12 & 0.42 & -54.97 \\
\hline
\end{tabular}

Ion implantation was performed in Ion Beam laboratory of Slovak University of Technology (STU), Bratislava. The basic instrument of the laboratory is a self-constructed $1 \mathrm{MV}$ open-air cascade accelerator. A displacement-per-atom (dpa) parameter was calculated in order to characterise the radiation damage; in Table 2 more details of the proton irradiation are given.

Table 2: Characteristics of ion-implantation experiments and calculated displacement-per-atom (dpa) parameter for irradiated samples.

\begin{tabular}{|c|c|c|c|c|}
\hline \multirow{3}{*}{$\begin{array}{l}\text { Sample } \\
\text { MA-635 }\end{array}$} & \multirow{2}{*}{\multicolumn{2}{|c|}{$\begin{array}{c}\mathrm{E}=160 \mathrm{keV}, \mathrm{T}<100^{\circ} \mathrm{C} \\
\mathrm{Q}_{1} \\
\mathrm{dpa}_{1} \\
{\left[\mathrm{C} / \mathrm{cm}^{2}\right]}\end{array}$}} & \multicolumn{2}{|c|}{$\mathrm{E}=415 \mathrm{keV}, \mathrm{T}<100^{\circ} \mathrm{C}$} \\
\hline & & & $\mathrm{Q}_{2}\left[\mathrm{C} / \mathrm{cm}^{2}\right.$ & dpa $_{2}$ \\
\hline & 0.569 & 0.0086 & 0.160 & 0.0032 \\
\hline $\mathrm{MA}-440$ & 0.512 & 0.0073 & 0.170 & 0.0034 \\
\hline MA-175 & 0.516 & 0.0078 & 0.165 & 0.0033 \\
\hline
\end{tabular}

The hardness measurements were done using a Shimadzu HSV-20 instrument at the AMES laboratory of JRC Petten. The positron annihilation spectroscopy (PAS) is used for nondestructive indirect investigation of materials. This method was applied in investigation of the RPV model alloys at the STU's Department of Nuclear Physics and Technology.

The results of hardness and thermoelectric measurements are summarised in Table 3. A significant decrease of hardness with dose (dpa) was observed and this phenomenon could be assigned to the nickel effect, which is responsible for softening process in steels. From these outcomes we can assume that nickel is stabilizing the matrix at increasing level of dose (dpa).

Table 3: Hardness parameters (HV0.5, HV1 and HV10) and STEAM of RPV model alloys.

\begin{tabular}{|c|c|c|c|c|c|}
\hline Sample & mdpa & HV0.5 & HV1 & HV10 & $\begin{array}{c}\text { RSC } \\
\mu \text { V/ }\end{array}$ \\
\hline $\begin{array}{c}\text { MA-635 } \\
\text { (Ni) } 0.007 w t \%\end{array}$ & 0 & 191 & 185 & 115 & 1.418 \\
\hline $\begin{array}{c}\text { MA-440 } \\
\text { (Ni) } 0.71 w t \%\end{array}$ & 8.6 & 158 & 158 & 105 & 1.684 \\
\hline $\begin{array}{c}\text { MA-175 } \\
\text { (Ni) } 1.14 w t \%\end{array}$ & 0 & 146 & 117 & 107 & - \\
\hline
\end{tabular}


In the Fig. 4 it can be observed how the RSC values are increasing with the dose (mdpa) as it occurs and it is systematically observed for the model alloys that were neutron irradiated. The increase is anyhow rather small and almost independent on the different chemical composition. Probably this is due to the fact that the irradiation was carried out at very high lead factor and at such relative low temperature that the effect of impurities is negligible (diffusivity and time for diffusion are extremely low). In this case what is observed by STEAM technique is a change due to direct matrix damage at the irradiation temperature. Data at higher dose would be very important and an experimental programme is ongoing in co-operation with STU.

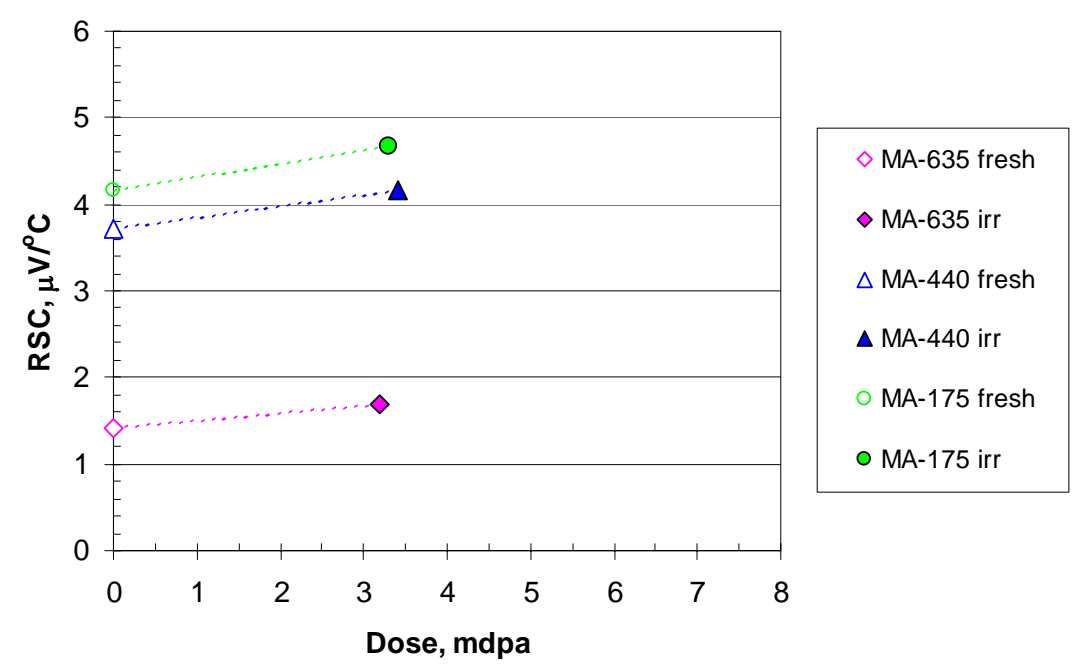

Fig. 4: The change of RSC with dose (dpa).

The PAS lifetime (LT) measurements of non-irradiated and irradiated samples were performed using conventional 2-detectors technique. The positron source $\left(\sim 1 \mathrm{MBq}\right.$ of ${ }^{22} \mathrm{Na}$ encapsulated into a kapton foil of $7.5 \mu \mathrm{m}$ thickness) was sandwiched between two plates of the investigated steel. At least $5-10 \times 10^{6}$ counts were collected for each spectrum. Measured spectra were decomposed and evaluated by using the dedicated software LT9 [11].

To evaluate the PAS results the mean-lifetime (MLT) and two-components analyses were applied; see Table 4. For the general interpretation, it can be considered that the positron lifetime (PL) technique is a well-established method for studying open-volume type atomic defects and defect impurity interactions in metals and alloys. The lifetime of positrons trapped at vacancies, vacancy-impurity pairs, dislocations, micro-voids, etc. is longer than that of free positrons in the perfect region of the same material. As a result of the presence of open-volume defects, the average positron lifetime observed in structural materials is found to increase with damage $[12,13]$.

Table 4: PAS LT parameters of RPV model alloys for different (p) implantation levels.

\begin{tabular}{|c|c|c|c|c|c|c|c|}
\hline Sample & mdpa & $\tau_{1}$ (ps) & $I_{1}(\%)$ & $\tau_{2}(p s)$ & $I_{2}(\%)$ & $V F$ & $\begin{array}{c}\text { MLT } \\
\text { (ps) }\end{array}$ \\
\hline \multirow{3}{*}{$\begin{array}{c}\text { MA-635 } \\
(\mathrm{Ni}) \\
0.007 \mathrm{wt} \%\end{array}$} & 0 & 104 & 75.4 & 216 & 24.6 & 1.31 & 131.6 \\
\hline & 3.2 & 117 & 79.9 & 271 & 20.1 & 1.53 & 150.0 \\
\hline & 8.6 & 109 & 85.5 & 288 & 13.5 & 1.21 & 151.9 \\
\hline \multirow{3}{*}{$\begin{array}{c}\text { MA-440 } \\
\text { (Ni) } 0.71 \mathrm{wt} \%\end{array}$} & 0 & 114 & 82.4 & 225 & 16.9 & 1.15 & 142.2 \\
\hline & 3.4 & 116 & 84.1 & 310 & 15.9 & 1.23 & 147.0 \\
\hline & 7.3 & 119 & 90.1 & 352 & 9.4 & 0.99 & 153.0 \\
\hline \multirow{3}{*}{$\begin{array}{c}\text { MA-175 } \\
\text { (Ni) } 1.14 \mathrm{wt} \%\end{array}$} & 0 & 114 & 78.9 & 170 & 20.6 & 1.29 & 136.9 \\
\hline & 3.3 & 115 & 82.1 & 299 & 17.9 & 1.28 & 149.0 \\
\hline & 7.8 & 119 & 86.8 & 277 & 11.9 & 1.01 & 155.1 \\
\hline
\end{tabular}


Lifetime $\tau_{1}$ of about $110 \mathrm{ps}$ is the dominant low-alloyed steel component with an intensity $\mathrm{I}_{1}$ of about $80 \%$. This component is assigned to the bulk properties slightly influenced via small defects. The second component $\tau_{2}$ with intensity $\mathrm{I}_{2}<20-25 \%$ and value about 220-350 ps can be assigned to the contribution of large vacancy type defects and/or small vacancy clusters.

It was found that the positron mean lifetime increase with $\mathrm{Ni}$ and $\mathrm{Cu}$ content. The proton bombardment caused the increasing of MLT parameter with the (dpa) level due to primaryknocked-atoms movement (Fig. 5a). The most significant increase was detected at the first implantation level $(\sim 3 \mathrm{mdpa})$. According to the intensities of the two dominant lifetime components, it seems to be systematical, that larger defects agglomerations are created after proton bombardment and their density decreases. An increase of MLT values to about 150 ps after implantation can hint to higher annihilation in defects concentrated at dislocation lines. As it can be seen, the MLT parameter is decreasing significantly with increasing of hardness HV (Fig. 5b).
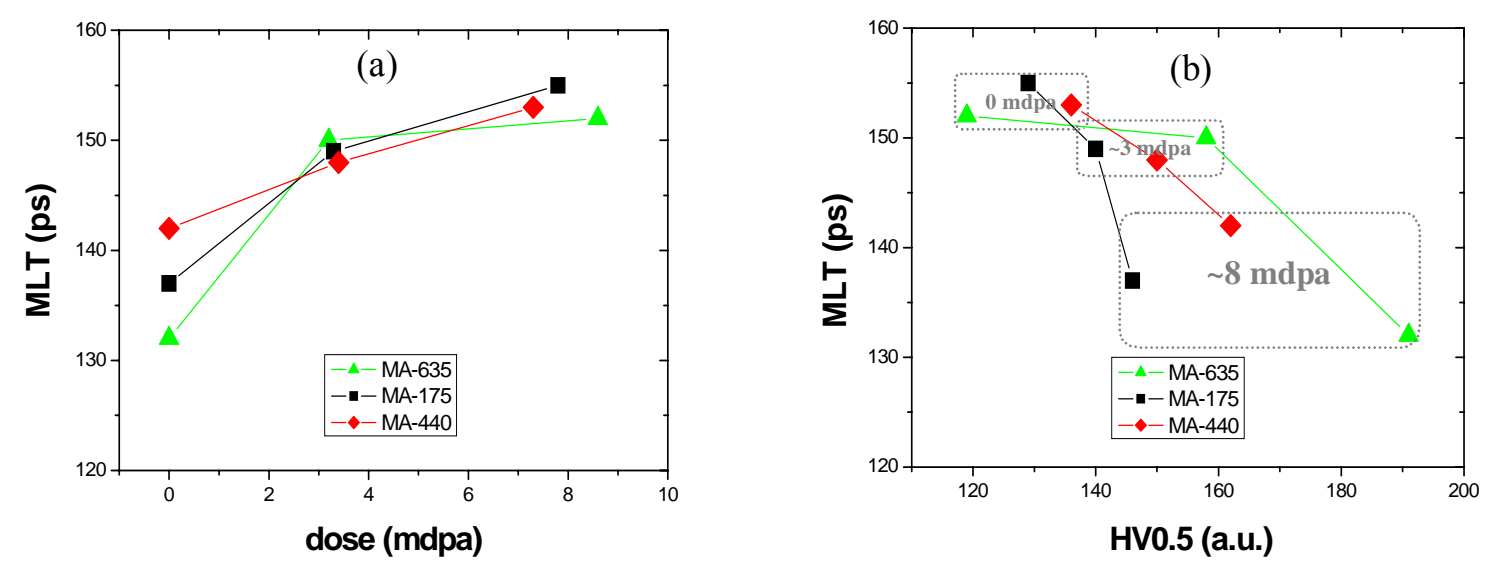

Fig. 5: (a) The change of MLT parameter with dose (dpa),

(b) The change of MLT parameter with hardness (HV).

The MA-175 damage mechanism can be considered to be a priory dominated by the direct matrix damage. This conclusion can be drawn by the fact that the amount of copper and phosphorus are rather low and nickel is known to enhance matrix damage. Material damage due to direct matrix damage, expressed as the transition temperature shift, is normally described as a power $1 / 2$ of the damage parameter [14]. By plotting the increase of MLT for MA-175, we can see that indeed, a power $1 / 2$ function of the dose follows very well the available data, indicating that the MLT shift could be related to matrix damage; see Fig. 6.

The situation is rather different if we consider MA-635. Here the damage is probably dominated by the precipitation/segregation of phosphorus present in rather large quantity; while copper content is negligible. This is also corroborated by the RSC measurements. Since the amount of precipitating/segregating elements $(\mathrm{P})$ is finite in the matrix, the process is saturating as soon as phosphorus matrix depletion occurs. Such saturation behaviour of the low Ni model alloys damage have been observed and confirmed recently as well as the non-saturation of the high Ni model alloys damage $[5,6]$. Surprisingly, the MLT increase seems again to be a good qualitative indicator of the process kinetic.

To be mentioned is the fact that by neutron irradiation MA-175 resulted much more sensitive to neutron radiation than MA-635 (almost the double shift on DBTT was obtained) [5,14].

The results for MA-440, where the expected damage is mainly copper precipitation enhanced by nickel, in combination with significant matrix damage, are unexpectedly not showing any saturating trend. On the contrary, the derived power function is even rather in excess of the power $1 / 2$ curve. The increase of MLT is anyhow smaller than in previous alloys in spite of the 
fact that the alloy resulted the most sensitive of the three alloys to radiation embrittlement when irradiated by neutrons.

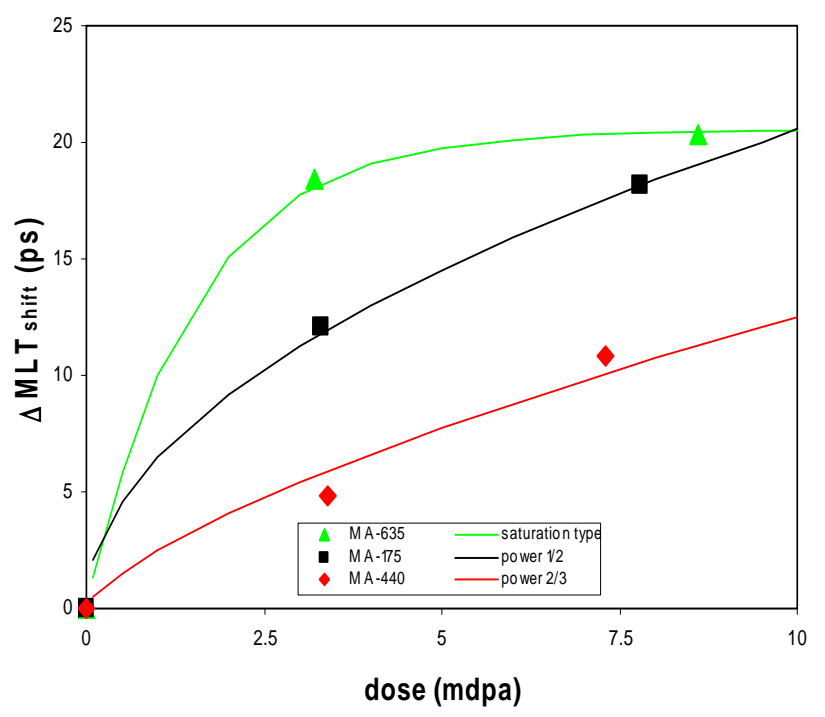

Fig. 6: The shift of MLT parameter with dose (dpa).

It seems that the MTL increase is sensible to specific parts of the damage; and not very much on copper induced damage. The results are very interesting but based only on data for 3 selected RPV model alloys. More systematic studies need to be done to clarify to which extent the increase of MLT is related quantitatively to the various damage contributions.

\section{Conclusions}

The results of this experimental work offered a unique opportunity for comparison and for studying the existing correlation between destructive and non-destructive outcomes for RPV model alloys in as-received condition and at different types and levels of irradiation. Similarly to neutrons, displacement (direct matrix damage) and precipitation (impurities like copper) are supposed to be the dominant irradiation degradation mechanisms generated by proton bombardment. Significant changes due to different irradiation levels were observed with using of PAS technique. Meaningful influence of alloying elements to irradiation degradation process of microstructure was identified by analysis of mean-lifetime (MLT) parameter as well as an interesting interdependency of positron MLT parameter and hardness HV. The effect of hardness decreasing resulted most significant for samples with low nickel content and from this fact we can conclude that nickel is stabilizing the matrix at increasing proton damage (dpa).

The results obtained from the measurement of the thermoelectric voltage are encouraging, showing that the STEAM non-destructive method has very high potential for studying the copper precipitation contribution in the radiation embrittlement phenomenon.

Finally, the combination of various techniques, based on macro and microstructure assessment, the RPV degradation process and its prediction will be necessary for deeper understanding of degradation mechanisms.

\section{References}

[1] Odette G.R.: On the dominant mechanism of irradiation embrittlement of reactor pressure vessel steels, Scripta Metallurgica Volume 17, Issue 10, 1983, 1183-1188. 
[2] Phythian W.J., English C.A.: Microstructural evolution in reactor pressure vessel steels, Journal of Nuclear Materials, Volume 205, 1993, 162-177.

[3] Slugen V., Zeman A., Lipka J., Debarberis L.: Positron annihilation and Mössbauer spectroscopy applied to WWER-1000 RPV steels in the frame of IAEA High Ni Coordinated Research Programme, NDT \& E International, Volume 37, Issue 8, 2004, 651661.

[4] Davies L.M.: A comparison of Western and Eastern nuclear reactor pressure vessel steels, International Journal of Pressure Vessels and Piping, Volume 76, Issue 3, 1999, 163-208.

[5] Debarberis L., Sevini F., Acosta B., Kryukov A., Erak D.: Fluence rate effects on irradiation embrittlement of model alloys, International Journal of Pressure Vessels and Piping, Volume 82, Issue 5, 2005, 373-378.

[6] Debarberis L., Acosta B., Sevini F., Kryukov A., Gillemot F., Valo M., Nikolaev A., Brumovsky M.: Role of nickel in a semi-mechanistic analytical model for radiation embrittlement of model alloys, Journal of Nuclear Materials, Volume 336, Issues 2-3, 2005, 210-216.

[7] Nikolaev Y. A., Nikolaeva A.V., Kryukov A.M., Shtrombakh Y.I., Platonov P.A.: Proceedings of the TACIS Workshop on the RPV Life Predictions, PCP3-ENUKRA-D4, 2000.

[8] Kohopaa J., Ahlstrand R.: Re-embrittlement behaviour of VVER-440 reactor pressure vessel weld material after annealing, International Journal of Pressure Vessels and Piping, Volume 77, Issue 10, 2000, 575-584.

[9] Debarberis L., Acosta B., Sevini F., Pirfo S., Hyde J. M., Hutchings M. T., Ortner S.: Studies of radiation embrittlement of model alloys by positron annihilation, thermo-electric and magnetic measurements, NDT \& E International, Volume 37, Issue 1, 2004, 19-22.

[10] Acosta B., Debarberis L., Sevini F., Kryukov A.: Cu effects on radiation embrittlement of Ni-containing complex model alloys and the related potentials of the thermoelectric method, NDT \& E International, Volume 37, Issue 4, 2004, 321-324.

[11] Kansy J., Microcromputer program for analysis of positron annihilation lifetime spectra, Nuclear Instruments and Methods in Physics Research Section A: Accelerators, Spectrometers, Detectors and Associated Equipment, Volume 374, Issue 2, 1996, 235-244.

[12] Valo M., Krause R., Saarinen K., Hautojärvi P. Hawthorne R.,: Irradiation response and annealing behaviour of pressure vessel model steels and iron ternary alloys measured with positron techniques, ASTM STP 1125, R.E. Stoller, Philadelphia, 1992, 172-185.

[13] Kočík J., Keilová E., Č́ižek J., Procházka I.: TEM and PAS study of neutron irradiated VVER-type RPV steels, Journal of Nuclear Materials, Volume 303, Issue 1, 2002 52-64.

[14] Debarberis L., Kryukov A., Gillemot F., Acosta B., Sevini F.: Semi-mechanistic analytical model for radiation embrittlement and re-embrittlement data analysis, International Journal of Pressure Vessels and Piping, Volume 82, Issue 5, 2005, 195-200. 